\title{
Rotación de residentes de medicina familiar en Unidad de Investigación Epidemiológica y en Servicios de Salud (experiencia de cinco años)
}

\author{
E. Villarreal-Ríos a , J. Velázquez-Tlapanco b , E.R. Vargas-Dazaa , L. Martínez-Martínez b' \\ L. Martínez-González ${ }^{a}$, L. Galicia-Rodrígueza , M.R. Ruelas-Candelas ${ }^{\text {b }}$
}

Introducción. Se presenta la experiencia de cinco años del Programa de Rotación del Residente de Medicina Familiar en la Unidad de Investigación. Materiales y métodos. El programa fue diseñado por los investigadores de la Unidad de Investigación Epidemiológica y en Servicios de Salud Querétaro, México, y los profesores de la Residencia de Medicina Familiar de la Unidad de Medicina Familiar n. ${ }^{\circ}$ 16. Durante el segundo año de residencia, se estableció la rotación por la unidad de investigación con una duración de dos meses, en la que se incluyó el desarrollo de un proyecto de investigación operativa. El programa de trabajo incluyó los contenidos teóricos de metodología de investigación cursados durante el primer año de residencia. La difusión se realizó al personal directivo en forma oral y por escrito, con la elaboración de la síntesis ejecutiva y del artículo científico. Resultados. Después de cinco años y cuatro meses, han asistido 40 médicos residentes. Se han desarrollado 39 protocolos de investigación, 23 seminarios, 39 síntesis ejecutivas, dos artículos publicados, un libro con 16 capítulos y otro en prensa. Las presentaciones en congresos incluyeron 34 delegacionales, 16 regionales y 7 nacionales. Conclusión. A cinco años de implementado el Programa de Rotación de Residente de Medicina Familiar en Unidad de Investigación, se puede afirmar que es productivo. Los resultados a corto y medio plazo así lo demuestran.

Palabras clave. Formación. Investigación. Residente.

\section{Clerkship of family medicine residents in a Unit of Epidemiological Research (five years experience)}

Introduction. The proposal is to present five years experience of Family Medicine Student in the Research Unit. Materials and methods. Program was designed by Researchers of
Epidemiological and Health Services Unit of Querétaro, México and Professors of Family Medicine Residence No 16. Rotation was specified for the second year of the Residence, duration was defined in two months, included theoretical and practical aspect, an operative research project was a requirement. The program contemplated oral presentation to directive personnel and executive synthesis. Results. After five years and four months, 40 Medical Residents have rotated, 39 whit research projects, 23 academic sessions, 39 executive synthesis, two papers, one book, 16 books chapters and one book in press, 34 presentations in local congress, 16 regional and seven national. Conclusion. After five years of Family Medicine Student in research unit, it is possible to affirm that it is productive. Short and medium term results therefore demonstrate.

Key words. Education. Research. Resident.

\section{Introducción}

En México, como parte del programa de formación de recursos humanos, el Instituto Mexicano del Seguro Social (IMSS), en coordinación con Universidades del país, diseñó e implementó el programa académico de especialización en Medicina Familiar, el mismo que es instrumentado por la Coordinación de Educación Médica de la propia institución. El programa se divide en tres áreas: técnico-médica, técnico-administrativa y de educación e investigación; a su vez, se subdividen en módulos con unidades didácticas que propician la revisión teórica [1].

El plan de estudios se desarrolla en la Unidad de Medicina Familiar, por un período de 3 años;

\footnotetext{
a Unidad de Investigación Epidemiológica y en Servicios de Salud de Querétaro.

b Residencia de Medicina Familiar de la Unidad de Medicina Familiar n. 16 de Querétaro.
}

Instituto Mexicano del Seguro Social. Querétaro, Querétaro de Arteaga, México.

\section{Correspondencia}

Dr. Enrique Villarreal Ríos. Unidad de Investigación Epidemiológica y en Servicios de Salud. Instituto Mexicano del Seguro Social. Zaragoza y 5 de Febrero. Col. Centro. Querétaro, Querétaro de Arteaga, México. CP. 76000.

Fax

(442) 2112300

E-mail enrique.villarreal@imss. gob.mx

Este trabajo fue posible gracias a la visión del Dr. Alberto Mejía Damián, jefe de Prestaciones Médicas en la Delegación Querétaro, México, quien apoyó la propuesta inicial y propició los espacios para las actividades académicas con el Cuerpo Directivo Delegacional.

Aceptación definitiva 08.05.2008. 
durante este tiempo, se realizan rotaciones complementarias en el Hospital General de Zona y la Unidad Rural de Campo del Sistema IMSSSolidaridad, para la adquisición de habilidades prácticas en aspectos técnico-médico y técnicoadministrativo [1]. En el área de investigación, se propuso la rotación del residente de Medicina Familiar por la Unidad de Investigación Epidemiológica y en Servicios de Salud Querétaro, México, para el desarrollo de habilidades en el área de investigación.

En la bibliografía existen trabajos que con diferente modalidad incorporan la investigación al proceso de formación del médico residente; sin embargo, no existe evidencia de resultados [2-5].

En este contexto, el objetivo del trabajo es presentar la experiencia de cinco años del Programa de Rotación de residentes de Medicina Familiar en la Unidad de Investigación.

\section{Materiales y métodos}

\section{Diseño del programa}

La propuesta se originó en la inquietud de los investigadores de la Unidad de Investigación Epidemiológica y en Servicios de Salud de Querétaro, México, y los profesores de la Residencia de Medicina Familiar de la Unidad de Medicina Familiar n. ${ }^{\circ} 16$ de la Ciudad de Querétaro, por encontrar un campo práctico para el desarrollo de los componentes teóricos del módulo de investigación, parte integral de la carga académica del Programa de Residencia.

Se efectuaron reuniones de intercambio conceptual para establecer el objetivo que se pretendía alcanzar y definir el perfil de ingreso y egreso del residente en la Unidad de Investigación, así como elaborar el programa de rotación. Como requisitos de ingreso se establecieron: cursar y aprobar los módulos y contenidos teóricos de metodología y bioestadística, durante el primer año del programa. La rotación por la Unidad de Investigación se diseñó para el segundo año con las obligaciones y beneficios propios de un investigador en Unidad de Investigación. El perfil de egreso incluyó plantear y desarrollar un proyecto de investigación [6]. Paralelamente, los investigadores de la Unidad de Investigación elaboraron el Programa de Rotación [7].
Inicialmente, el tiempo de la rotación se estableció para un mes y posteriormente se extendió a dos, durante el cual se desarrolló un proyecto de investigación operativa $[7,8]$, a partir de un problema cotidiano de la práctica médica y con la posibilidad de utilizar las bases de datos institucionales. El programa incluyó la difusión de los resultados a los directivos de la Delegación tanto en presentación oral [7,9] en formato de seminario como en difusión escrita $[7,10]$ en síntesis ejecutiva, a la que posteriormente se añadió el artículo científico.

\section{Implementación del programa}

El programa [4] fue aprobado por la Dirección de la Unidad de Medicina Familiar sede de la Residencia, así como por la Coordinación de Educación de la Delegación y la Jefatura de Prestaciones Médicas Delegacional, para iniciarse en marzo de 2002.

\section{Propósito del programa de rotación}

Se planteó como propósito de la rotación del residente de Medicina Familiar en la Unidad de Investigación Epidemiológica y en Servicios de Salud Querétaro, México, integrar los elementos teóricos con la práctica a través del proceso de investigación.

\section{Desarrollo operativo del programa}

Las actividades del Programa de Rotación [11] se realizaron bajo la modalidad tutorial con espacios de reflexión para el residente:

- Se inician con el planteamiento de la pregunta de investigación y la descripción del problema; al final de la primera etapa se cuenta con un documento que incluye, además de lo anterior, el marco teórico, los objetivos y las hipótesis de investigación.

- La metodología y el formato de recolección de información se desarrollan durante la segunda etapa.

- En la tercera, se recoge la información y se construye la base de datos.

- En el transcurso de la cuarta etapa se realiza el análisis de los datos, la redacción y discusión de los resultados, y se integran tanto el formato de síntesis ejecutiva como el artículo científico. 
- En la quinta etapa, el residente elabora una presentación oral de los resultados durante un seminario académico al que se invita a las autoridades de la Institución, quienes reciben en formato de síntesis ejecutiva el trabajo realizado.

\section{Evaluación del programa}

La evaluación y el seguimiento del programa se planearon a corto, medio y largo plazos. A corto plazo incluye la presentación de resultados a directivos de la Delegación e información escrita en formato de síntesis ejecutiva. A medio plazo, se contempla la participación en congresos y la publicación en revistas científicas. A largo plazo, la evaluación incluye la elaboración de proyectos de investigación como médico de familia, y la realización del seguimiento de la influencia de la rotación en el quehacer cotidiano mediante la incorporación del método científico al método clínico.

\section{Resultados}

Después de cinco años y cuatro meses de haberse iniciado el programa, han rotado por la Unidad de Investigación 40 residentes, 39 han completado la estancia y en una ocasión se ha suspendido por no reunir el perfil necesario. En todos los casos se plantearon protocolos [12] de investigación relacionados con las líneas de investigación definidas, diabetes mellitus, perfil de uso de servicios, coste de la atención, uso de tecnología y paciente geriátrico.

Se efectuaron 23 seminarios académicos [13] con directivos de la Delegación, durante los cuales el residente responsable de la investigación realizó la presentación oral de la metodología utilizada y de los resultados encontrados -la actividad incluyó tiempo de exposición, preguntas y respuestas-, y en 39 casos se redactaron síntesis ejecutivas [14].

Se elaboraron 13 artículos científicos; de ellos, ocho se encuentran en proceso de evaluación por algún comité editorial y se han publicado dos $[15,16]$. Existe un libro publicado [17] de 16 capítulos (El sistema de salud y el paciente con diabetes) y se encuentra en prensa un segundo libro (El sistema de salud, el perfil de uso y el costo de la atención).

Se participó con 34 presentaciones en Reuniones Estatales de Investigación [18-24], 16 en
Reuniones Regionales [25-31] y siete en Reuniones Nacionales [32-36].

Como parte de la evaluación, se midió el nivel de satisfacción del residente en relación con el programa de rotación, se incluyeron siete rubros (objetivos, metodología, aprendizaje, enseñanza, recursos, evaluación e impacto), se utilizó la escala de Likert (muy buena, buena, regular, mala y muy mala satisfacción). La encuesta fue aplicada por los profesores de la residencia un día específico en el área de enseñanza de la sede de la residencia (Unidad de Medicina Familiar). El resultado indica que el nivel de satisfacción muy buena predominó, con porcentajes del 60-100\% en los rubros evaluados (Tabla).

\section{Discusión}

Cuando se plantean contenidos teóricos de investigación como parte del programa de formación del residente de Medicina Familiar y, en este caso, la rotación por la Unidad de Investigación, el objetivo no es formar al residente como investigador, así como tampoco se pretende que el residente de Medicina Familiar se convierta en cirujano o pediatra al rotar por estos servicios. Los programas de maestrías y doctorados se encargan de cumplir con ello.

El propósito de integrar aspectos teóricos de investigación con los prácticos en la formación del residente es realizar un acercamiento del médico en formación al método científico, para llevarlo al terreno de la práctica clínica cotidiana.

La investigación, tradicionalmente, se ha concebido como un proceso largo, del cual se obtienen resultados después de uno o dos años y, en ocasiones, más tiempo. Esto propicia una de las grandes críticas que se le pueden hacer a la propuesta aquí planteada, 'el corto tiempo programado para desarrollar el trabajo de investigación', inicialmente de un mes y, posteriormente, por ser insuficiente, modificado a dos meses.

En defensa de ello, es menester señalar la importancia de la capacitación teórica desarrollada durante el primer año de la residencia, la cual favorece que durante el transcurso de la actividad práctica en la Unidad de Investigación, el residente cuente con un marco teórico que le permite desarrollar sus actividades, facilitando la modalidad tutorial con espacios para la reflexión 
Tabla. Nivel de satisfacción del residente por elemento evaluado con relación al programa de rotación.

\begin{tabular}{lccccc}
\hline & Muy buena & Buena & Regular & Mala & Muy mala \\
\hline Objetivos & $60 \%$ & $40 \%$ & - & - & - \\
\hline Metodología & $60 \%$ & $30 \%$ & $10 \%$ & - & - \\
\hline Aprendizaje & $100 \%$ & - & - & - & - \\
\hline Enseñanza & $100 \%$ & - & - & - & - \\
\hline Recursos & $70 \%$ & $20 \%$ & - & $10 \%$ & - \\
\hline Evaluación & $70 \%$ & $30 \%$ & - & - & - \\
\hline
\end{tabular}

y discusión con el investigador en cada uno de los temas abordados.

Aunado a ello, es menester señalar que trabajar con bases de datos existentes en la institución, además de acortar el tiempo, se encuentra utilidad práctica a un cúmulo de información almacenada y se ofrece respuesta a un problema operativo del sistema de salud; ésta es una de las características de la investigación operativa: ofrecer respuestas en un tiempo corto. No obstante, deberá reconocerse que ello es factible en la investigación epidemiológica y en sistemas de salud, a diferencia de la investigación clínica y biomédica, la cual difícilmente puede efectuarse a partir de la explotación de bases de datos, dado su carácter eminentemente experimental, aunque esto no es imposible.

La presentación de los resultados de la investigación realizada por el residente en seminarios académicos integrado por auditorios de directivos, tiene como propósito vincular la investigación con la parte asistencial de las instituciones de salud. Si aunado a esto el problema de investigación es operativo, entonces este lazo se estrecha. La intención final es presentar al directivo un panorama del problema estudiado y propiciar el análisis de los resultados, buscando con ello que sean contemplados en el proceso de toma de decisiones; esta es una actividad eminentemente directiva, por lo que el investigador deberá mantener la objetividad. Lo cual no lo exime de la obligación de proporcionar el análisis del problema desde una perspectiva científica y ofrecer posibles soluciones con la intención de que los resultados de la investigación sean utilizados.

El directivo no es el único usuario de los resultados de investigación; en realidad, es un personaje que ha surgido recientemente. Es la comunidad científica el usuario original, perspectiva que debe integrar el residente a través de la redacción del artículo científico que potencialmente pueda publicarse. No obstante, los resultados obtenidos marcan una desproporción entre las síntesis ejecutivas elaboradas y los artículos publicados; esto se debe a que la orientación inicial de la rotación se enfocaba a la presentación de resultados a directivos, situación que se ha reorientado hacia la búsqueda de la publicación científica, sin perder con ello el contacto con el directivo.

Las presentaciones en congresos se dan en esta misma línea; son foros en los cuales la discusión académica permite que el residente explique los problemas desde la lógica del método científico, y probablemente esto no se puede asegurar con la información aquí presentada, y que utilice esa misma lógica durante el acto de la práctica médica.

Existen otras propuestas de capacitación en investigación para el médico residente clínico y quirúrgico; en ellas, el objetivo no es complementar la formación como médico clínico [2]; se percibe una tendencia a formar investigadores. Los programas de Geriatría, Medicina Preventiva y Salud Pública también incorporan la investigación a sus programas, pero no como una rotación formal por la unidad de investigación; la propuesta se centra en cursos teóricos, el acer- 
camiento con los investigadores y la realización de proyectos de investigación [3-5]. Independientemente de las modalidades planteadas, no existen publicaciones donde se presenten los resultados obtenidos.

Es necesario reconocer que queda pendiente la evaluación a largo plazo; no obstante, a cinco años de implementado el Programa de Rotación de Residente de Medicina Familiar en Unidad de Investigación, se puede decir que es productiva. Los resultados a corto y medio plazo así lo demuestran. La evaluación a largo plazo deberá identificar el tipo de proyectos realizados, el impacto de los resultados de investigación desde la perspectiva académica y el uso de resultados, sin olvidar que la esencia del médico es la atención al paciente, aspecto éste sobre el que debe impactar la formación en investigación.

\section{Bibliografía}

1. Programa Académico del Curso de Especialización en Medicina Familiar. Dirección de Prestaciones Médicas. Coordinación de Educación Médica. México DF: Instituto Mexicano del Seguro Social; 2000.

2. Programa tutelar de investigación en salud para Médicos Residentes (DF). URL: http://edumed.imss.gob.mx/ eventos/Ponencias/Programa\%20Tutelar\%20de\%20Investigacion $\% 20$ en $\% 20$ Salud\%20para\%20Medicos\%20 Residentes\%20(DF).ppt. [22.08.2007].

3. Formación en Geriatría. Hospital Comarcal Sant Antoni Abat. URL: http://fhcsaa.cat/e50dr.htm. [30.01.2008].

4. Programa de rotaciones de los residentes de Medicina Preventiva y Salud Pública en el complejo Hospitalario Universitario de Albacete. URL: www.chospab.es/area_ medica/medicinapreventiva/ProgramaresidenciaMedicinaPreventiva.doc. [30.01.2008].

5. Residencia en Medicina Preventiva General, Centro Médico Wyckoff Heights, Centro Médico Mount Sinai. URL: http://wyckoffhospital.drtango.com/intern_prevent_ edu.htm. [30.01.2008].

6. Convenio de colaboración entre la Unidad de Investigación Epidemiológica y en Servicios de Salud Querétaro y la Jefatura de Educación Médica de la Unidad de Medicina Familiar No 16 Querétaro Sede de la Residencia. Querétaro de Arteaga; 2002.

7. Programa de Rotación de Residentes de Medicina Familiar en Unidad de Investigación Epidemiológica y en Servicios de Salud Querétaro. México DF: Instituto Mexicano del Seguro Social; 2002.
8. Lineamientos para la elaboración de Protocolos de Investigación, Unidad de Investigación Epidemiológica y en Servicios de Salud Querétaro. México DF: Instituto Mexicano del Seguro Social; 2002.

9. Lineamientos para la elaboración de Presentación Oral de Resultados. Unidad de Investigación Epidemiológica y en Servicios de Salud Querétaro. México DF: Instituto Mexicano del Seguro Social; 2002.

10. Lineamientos para la elaboración de Síntesis Ejecutivas. Unidad de Investigación Epidemiológica y en Servicios de Salud Querétaro. México DF: Instituto Mexicano del Seguro Social; 2002.

11. Cronograma de actividades del Programa de Rotación de Residentes de Medicina Familiar en la Unidad de Investigación Epidemiológica y en Servicios de Salud Querétaro. México DF: Instituto Mexicano del Seguro Social; 2002.

12. Acervo de Protocolos de Investigación del Programa de Rotación de Residentes de Medicina Familiar en la Unidad de Investigación Epidemiológica y en Servicios de Salud Querétaro, 2002-2006. Querétaro de Arteaga: UIESSQ; 2002.

13. Acervo de Presentaciones Orales del Programa de Rotación de Residentes de Medicina Familiar en la Unidad de Investigación Epidemiológica y en Servicios de Salud Querétaro 2002-2006. Querétaro de Arteaga: Querétaro de Arteaga: UIESSQ; 2002.

14. Acervo de Síntesis Ejecutivas del Programa de Rotación de Residentes de Medicina Familiar en la Unidad de Investigación Epidemiológica y en Servicios de Salud Querétaro, 2002-2006. Querétaro de Arteaga: UIESSQ; 2002.

15. Villarreal-Ríos E, Campos-Esparza M, Garza-Elizondo ME, Martínez-González L, Núñez-Rocha GM, RomeroIslas NR. Variación del costo unitario en una institución de seguridad social en Querétaro, México. Revista de Salud Pública 2006; 8: 153-62.

16. Villarreal-Ríos E, Campos-Esparza M, Romero-Islas NR, Garza-Elizondo ME, Martínez-González L, Cortés-Núñez AR. Coste de la atención al paciente diabético-hipertenso en primer nivel de atención. Atención Primaria 2006; 38: 537-43.

17. Villarreal-Ríos E, Vargas-Daza ER, Martínez-González L, Galicia-Rodríguez L. El sistema de salud y el paciente con diabetes. México DF: Instituto Mexicano del Seguro Social; 2005.

18. Compendio de trabajos de Investigación Reunión Delegacional del Instituto Mexicano del Seguro Social en Querétaro 2003. Querétaro de Arteaga: Instituto Mexicano del Seguro Social; 2003.

19. Memorias del Simposio CONCYTEQ 2003. Impulso a la Investigación en el Sector Salud. Querétaro, Querétaro de Arteaga; 2003.

20. I Congreso Estatal de Medicina Familiar. Sociedad de Mé- 
dicos Familiares de Querétaro. Querétaro, Querétaro de Arteaga; 2003.

21. Memorias Reunión Delegacional del Instituto Mexicano del Seguro Social Querétaro 2004. Querétaro de Arteaga: Instituto Mexicano del Seguro Social; 2004.

22. Memorias Reunión Delegacional del Instituto Mexicano del Seguro Social Querétaro 2005. Querétaro de Arteaga: Instituto Mexicano del Seguro Social; 2005.

23. Memorias Reunión Delegacional del Instituto Mexicano del Seguro Social Querétaro 2006. Querétaro de Arteaga: Instituto Mexicano del Seguro Social; 2006.

24. Memorias del Simposio CONCYTEQ 2006. Impulso a la Investigación en el Sector Salud. Querétaro, Querétaro de Arteaga; CONCYTEQ; 2003.

25. Memorias, VIII Foro Regional de Investigación en Salud, Región Centro. Querétaro, Querétaro de Arteaga: Instituto Mexicano del Seguro Social; 2002.

26. Memorias IX Foro Regional de Investigación en Salud, Región Centro. Ixtapa Zihuatanejo: Instituto Mexicano del seguro Social; 2003.

27. Memorias Foro Regional de Investigación en Salud, Región Occidente. Guanajuato, Guanajuato: Instituto Mexicano del Seguro Social; 2003.

28. Memorias Foro Regional de Investigación en Salud, Región Oriente. Playa del Carmen, Quintana Roo: Instituto Mexicano del Seguro Social; 2003.
29. Memorias X Foro Regional de Investigación en Salud, Región Centro. Veracruz: Instituto Mexicano del Seguro Social; 2004.

30. Memorias XI Foro Regional de Investigación en Salud, Región Centro. Ixtapan de la Sal: Instituto Mexicano del Seguro Social; 2005.

31. Memorias XII Foro Regional de Investigación en Salud, Región Centro. Querétaro de Arteaga: Instituto Mexicano del Seguro Social; 2006.

32. Memorias, XI Foro Nacional de Investigación en Salud, Coordinación de Investigación en Salud. Oaxtepec, Morelos: Instituto Mexicano del Seguro Social; 2002.

33. Memorias XII Foro Nacional de Investigación en Salud. Veracruz, Veracruz: Instituto Mexicano del Seguro Social; 2003.

34. Memorias XIII Foro Nacional de Investigación en Salud. Oaxtepec, Morelos: Instituto Mexicano del Seguro Social; 2004.

35. Memorias XIV Foro Nacional de Investigación en Salud. Oaxtepec, Morelos: Instituto Mexicano del Seguro Social; 2005.

36. Memorias XV Foro Nacional de Investigación en Salud. Oaxtepec, Morelos: Instituto Mexicano del Seguro Social; 2006. 